\title{
Supersymmetric Yang-Mills-Chern-Simons theory
}

F. Ruiz Ruiz ${ }^{\mathrm{a} *}$ and P. van Nieuwenhuizen ${ }^{\mathrm{b} \dagger}$

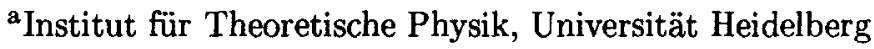

Philosophenweg 16, 69120 Heidelberg, Germany

b Institute for Theoretical Physics, State University of New York at Stony Brook

Stony Brook, NY 11794-3840, USA

We prove that three-dimensional $N=1$ supersymmetric Yang-Mills-Chern-Simons theory is finite to all loops. This leaves open the possibility that different regularization methods give different finite effective actions. We show that for this model dimensional regularization and regularization by dimensional reduction yield the same effective action.

\section{Introduction and conclusions}

The supersymmetric regularization of gauge theories remains a major unsolved problem in supersymmetry. In this contribution we consider supersymmetric Yang-Mills-Chern-Simons theory in three dimensions and prove that ordinary dimensional regularization (or DReG) [1] and regularization by dimensional reduction (or DReD) [2] preserve both supersymmetry and BRS invariance. We further show that they give the same Green functions. Our strategy is to first prove that the theory is finite to all loop orders, so that the regularized effective actions $\Gamma^{\mathrm{DReG}}$ and $\Gamma^{\mathrm{DReD}}$ are also renormalized effective actions and the difference $\Delta \Gamma=\Gamma^{\mathrm{DReG}}-\Gamma^{\mathrm{DReD}}$ is the difference of two renormalized effective actions. Next we show that this difference vanishes. This, together with the observations that DReG preserves at all stages the BRS identities of local gauge invariance and that $\mathrm{DReD}$ preserves supersymmetry, implies the thesis. We will also see that neither DReG nor DReD have problems in dealing with the $\epsilon^{\mu \nu \rho}$ that appears in the classical action of the model. The work reported here is based on ref. [3], to which we refer for more details.

\footnotetext{
*Alexander von Humboldt Fellow.

† Research supported by NSF grant Phy 9309888 .
}

\section{Superfields and components}

In three-dimensional $N=1$ supersymmetry, superspace is parameterized by three real spacetime coordinates $x^{\mu}$ and two real anticommuting Majorana spinor coordinates $\theta^{\alpha}$. Any vector $v^{\mu}$ can be represented as a symmetric rank-two vector $v^{\alpha \beta}$ with indices $\alpha, \beta=1,2$ through the relation $v_{\beta}^{\alpha}=\left(\gamma_{\mu}\right)_{\beta}^{\alpha} v^{\mu}$, where $\gamma_{\mu}$ are the Dirac matrices. The spinor superderivative $D_{\alpha}$ is defined by $D_{\alpha}=$ $\partial_{\alpha}+i \theta^{\beta} \partial_{\beta \alpha}$. Supersymmetry transformations are generated by the supercharge $Q_{\alpha}=\partial_{\alpha}-i \theta^{\beta} \partial_{\beta \alpha}$ and have the form $\delta x^{\alpha \beta}=a^{\alpha \beta}-2 i \varepsilon^{(\alpha} \theta^{\beta)}, \delta \theta^{a}=$ $\varepsilon^{\alpha}$, where $a^{\alpha \beta}$ is a real commuting constant vector, $\epsilon^{\alpha}$ is an anticommuting constant Majorana spinor and $\varepsilon^{(\alpha} \theta^{\beta)}=\frac{1}{2}\left(\varepsilon^{\alpha} \theta^{\beta}+\varepsilon^{\beta} \theta^{\alpha}\right)$. A superfield $\Psi(x, \theta)$ transforms linearly under the action of the supercharge: $\delta \Psi=\epsilon^{\alpha} Q_{\alpha} \Psi$.

The gauge field $A_{\alpha \beta}^{a}$ of a real, compact, semisimple Lie algebra with completely antisymmetric structure constants $f^{a b c}$ is part of a supermultiplet described by a Majorana spinor gauge potential $\Gamma_{\alpha}^{a}$ [4]. The superfield $\Gamma_{\alpha}^{a}$ defines a real vector gauge potential $\Gamma_{\alpha \beta}^{a}$ and an imaginary spinor field strength $W_{\alpha}^{a}$ through the equations

$$
\begin{aligned}
\Gamma_{\alpha \beta}^{a} & =D_{(\alpha} \Gamma_{\beta)}^{a}+\frac{i}{2} f^{a b c} \Gamma_{\alpha}^{b} \Gamma_{\beta}^{c} \\
W_{\alpha}^{a} & =D^{\beta} D_{\alpha} \Gamma_{\beta}^{a}+i f^{a b c} \Gamma^{b \beta} D_{\beta} \Gamma_{\alpha}^{c} \\
& -\frac{1}{3} f^{a b c} f^{c d e} \Gamma^{b \beta} \Gamma_{\beta}^{d} \Gamma_{\alpha}^{e} .
\end{aligned}
$$

Besides $A_{\alpha \beta}^{a}$, the supermultiplet contains a real 
scalar field $H^{a}$ and two anticommuting Majorana spinors $\chi_{\alpha}^{a}$ and $\lambda_{\alpha}^{a}$, given by the projections onto $\theta^{\alpha}=0$

$$
\begin{aligned}
\chi_{\alpha}^{a}=\Gamma_{\alpha}^{a} \mid & H^{a}=\frac{1}{2} D^{\alpha} \Gamma_{\alpha}^{a} \mid \\
A_{\alpha \beta}^{a}=\Gamma_{\alpha \beta}^{a} \mid & \lambda_{\alpha}^{a}=-\frac{i}{2} W_{\alpha}^{a} \mid .
\end{aligned}
$$

We will work in the supersymmetric Landau gauge, characterized by the condition $D^{\alpha} \Gamma_{\alpha}^{\alpha}=$ 0 . In this gauge, the classical $N=1$ Yang-MillsChern-Simons action has the form

$\Gamma_{0}=\frac{1}{m} S_{\mathrm{YM}}+S_{\mathrm{CS}}+S_{\mathrm{GF}}+S_{\mathrm{ES}}$,

where

$$
\begin{aligned}
S_{\mathrm{YM}} & =-\frac{1}{32 g^{2}} \int d^{3} x d^{2} \theta W^{a \alpha} W_{\alpha}^{a} \\
S_{\mathrm{CS}} & =\frac{i}{16 g^{2}} \int d^{3} x d^{2} \theta\left[\left(D^{\alpha} \Gamma^{a \beta}\right)\left(D_{\beta} \Gamma_{\alpha}^{a}\right)\right. \\
& +\frac{2 i}{3} f^{a b c} \Gamma^{a \alpha} \Gamma^{b \beta}\left(D_{\beta} \Gamma_{\alpha}^{c}\right) \\
& \left.-\frac{1}{6} f^{a b c} f^{c d e} \Gamma^{a \alpha} \Gamma^{b \beta} \Gamma_{\alpha}^{d} \Gamma_{\beta}^{e}\right] \\
S_{\mathrm{GF}} & =\frac{i}{4} \int d^{3} x d^{2} \theta s\left(\hat{C}^{a} D^{\alpha} \Gamma_{\alpha}^{a}\right)
\end{aligned}
$$

and

$$
S_{\mathrm{ES}}=\frac{i}{2} \int d^{3} x d^{2} \theta\left(\frac{1}{2} K_{\Gamma}^{a \alpha} s \Gamma_{\alpha}^{a}-K_{C}^{a} s C^{a}\right) .
$$

Here $m$ is a parameter with dimensions of mass, $g$ is a dimensionless coupling constant, $s$ is the BRS operator, $\hat{C}^{a}$ and $C^{a}$ are real anticommuting antighost and ghost superfields, and $K_{\Gamma}^{a \alpha}$ and $K_{C}^{a}$ are commuting external supersources coupled to the nonlinear BRS transforms $s \Gamma_{\alpha}^{a}$ and $s C^{a}$. The BRS transformations that leave $S_{\mathrm{YM}}, S_{\mathrm{CS}}$ and $S_{\mathrm{GF}}$ invariant are given by

$$
\begin{array}{ll}
s \Gamma_{\alpha}^{a}=i\left(\nabla_{\alpha} C\right)^{a} & s B^{a}=0 \\
s \hat{C}^{a}=B^{a} & s C^{a}=-\frac{1}{2} f^{a b c} C^{b} C^{c},
\end{array}
$$

with $\nabla_{\alpha}^{a b}=\delta^{a b} D_{\alpha}+i f^{a c b} \Gamma_{\alpha}^{c}$ the spinor covariant superderivative, $B^{a}$ a real Lagrange multiplier superfield imposing the gauge condition $D^{\alpha} \Gamma_{\alpha}^{a}=0$, and $s$ satisfying as usual $s^{2}=0$. The components of $B^{a}$ and $\hat{C}^{a}$ are defined by the projections

$$
\begin{array}{r|r}
b^{a}=B^{a} & \hat{c}^{a}=\hat{C}^{a} \\
\zeta_{\alpha}^{a}=i D_{\alpha} B^{a} & \hat{\varphi}_{\alpha}^{a}=D_{\alpha} \hat{C}^{a} \\
h^{a}=-\frac{i}{2} D^{2} B^{a} & \hat{\omega}^{a}=-\frac{i}{2} D^{2} \hat{C}^{a}
\end{array}
$$

those of $C^{a}$ by replacing hatted antighosts with unhatted ghosts, and those of $K_{\Gamma}^{a \alpha}$ and $K_{C}^{a}$ by

$$
\begin{array}{r|r}
\kappa_{\alpha}^{a}=K_{\alpha \Gamma}^{a} \mid & \ell^{a}=K_{C}^{a} \\
G^{a}=-\frac{i}{2} D^{\alpha} K_{\alpha \Gamma}^{a} \mid & \tau_{\alpha}^{a}=i D_{\alpha} K_{C}^{a} \\
K_{\alpha \beta}^{a}=i D_{(\alpha} K_{\beta) \Gamma}^{a} \mid & L^{a}=-\frac{i}{2} D^{2} K_{C}^{a} \\
\sigma_{\alpha}^{a}=-\frac{i}{2} D^{\beta} D_{\alpha} K_{\beta \Gamma}^{a} &
\end{array}
$$

By construction, $\Gamma_{0}$ is invariant under supersymmetry transformations.

To formulate DReG, we work with component fields. The terms $S_{\mathrm{CS}}, S_{\mathrm{YM}}, S_{\mathrm{GF}}$ and $S_{\mathrm{ES}}$ in $\Gamma_{0}$ are given in terms of components by

$$
\begin{aligned}
S_{\mathrm{YM}} & =\frac{1}{g^{2}} \int d^{3} x\left[-\frac{1}{4} F_{\mu \nu}^{a} F^{a \mu \nu}-\frac{1}{2} \bar{\lambda}^{a}(\not D \lambda)^{a}\right] \\
S_{\mathrm{CS}} & =\frac{1}{g^{2}} \int d^{3} x\left[\epsilon ^ { \mu \nu \rho } \left(\frac{1}{2} A_{\mu}^{a} \partial_{\nu} A_{\rho}^{a}\right.\right. \\
& \left.\left.+\frac{1}{6} f^{a b c} A_{\mu}^{a} A_{\nu}^{b} A_{\rho}^{c}\right)-\frac{1}{2} \bar{\lambda}^{a} \lambda^{a}\right] \\
S_{\mathrm{GF}} & =\int d^{3} x s\left(-\hat{c}^{a} \partial_{\mu} V^{a \mu}+i \overline{\hat{\varphi}}^{a} \Lambda^{a}-\hat{\omega}^{a} H^{a}\right),
\end{aligned}
$$

where $F_{\mu \nu}^{a}$ is the field strength, $D_{\mu}^{a b}=\delta^{a b} \partial_{\mu}+$ $f^{a c b} A_{\mu}^{c}$ is the covariant derivative, and

$$
\begin{aligned}
V_{\mu}^{a} & =A_{\mu}^{a}+\frac{1}{4} f^{a b c} \bar{\chi}^{b} \gamma_{\mu} \chi^{c} \\
\Lambda^{a} & =\lambda^{a}+\not \partial \chi^{a}+\frac{1}{2} f^{a b c} A^{b} \chi^{c}-\frac{1}{2} f^{a b c} H^{b} \chi^{c} \\
& -\frac{1}{24} f^{a b c} f^{c d e} \gamma^{\mu} \chi^{b}\left(\bar{\chi}^{d} \gamma_{\mu} \chi^{e}\right) .
\end{aligned}
$$

The action of $s$ on components is obtained from the definition of the latter as projections and the 
action of $s$ on superfields. It is given by

$$
\begin{aligned}
\Gamma_{\alpha}^{a}: \quad s \chi^{a}=i \varphi^{a}-f^{a b c} \chi^{b} c^{c} \\
s A_{\mu}^{a}=\left(D_{\mu} c\right)^{a} \\
s H^{a}=\omega^{a}+f^{a b c} H^{b} c^{c}-\frac{i}{2} f^{a b c} \bar{\chi}^{b} \varphi^{c} \\
s \lambda^{a}=-f^{a b c} \lambda^{b} c^{c} \\
B^{a}: \quad s b^{a}=0 \quad s \zeta^{a}=0 \quad s h^{a}=0 \\
\hat{C}^{a}: \quad s \hat{c}^{a}=b^{a} \quad s \hat{\varphi}^{a}=i \zeta \quad s \hat{\omega}^{a}=h^{a} \\
C^{A}: \quad s c^{a}=-\frac{1}{2} f^{a b c} c^{b} c^{c} \\
s \varphi^{a}=f^{a b c} \varphi^{b} c^{c} \\
s \omega^{a}=-f^{a b c} \omega^{b} c^{c}-\frac{1}{2} f^{a b c} \bar{\varphi}^{b} \varphi^{c} .
\end{aligned}
$$

The supersymmetry transformation laws for the components are obtained in the same way. Here we only present those for the components of the gauge multiplet:

$$
\begin{aligned}
& \delta \chi^{a}=N^{a} \varepsilon-H^{a} \varepsilon \\
& \delta A_{\mu}^{a}=\bar{\varepsilon} \gamma_{\mu} \lambda^{a}+\bar{\varepsilon}\left(D_{\mu} \chi\right)^{a} \\
& \delta H^{a}=-\bar{\varepsilon} \Lambda^{a} \\
& \delta \lambda^{a}=-\frac{1}{2} \gamma^{\mu} \gamma^{\nu} F_{\mu \nu}^{a} \varepsilon+f^{a b c} \lambda^{b}\left(\bar{\chi}^{c} \varepsilon\right) .
\end{aligned}
$$

Power counting shows that there is only a fi-

\begin{tabular}{|c|c|c|c|}
\hline \multicolumn{2}{|r|}{ external lines } & 2 loops & 3 loops \\
\hline$\chi \bar{\chi}$ & & $\bar{\omega}=1$ & $\bar{\omega}=0$ \\
\hline $\begin{array}{l}\bar{\lambda} \bar{\chi} \\
\chi \bar{\chi}\end{array}$ & $\begin{array}{lll}A^{2} & A H & H^{2} \\
\chi \bar{\chi} H & (\chi \bar{\chi})^{2} & \end{array}$ & $\bar{\omega}=0$ & \\
\hline
\end{tabular}
nite number of superficially divergent diagrams, thus proving that the theory is superrenormalizable. At one loop there are quadratic, linear and logarithmic divergences; at two loops there are linear and logarithmic divergences; and at three loops only logarithmic divergences survive. Furthermore, quadratically divergent one-loop diagrams do not have internal gauge lines and the only primitively divergent two and three-loop 1PI diagrams are those in Table 1 , where $\bar{\omega}$ denotes the superficial UV degree of divergence of the diagram.

Table 1: Power counting for component fields
The BRS identity for the full renormalized effective action $\Gamma$ takes the form

$$
\begin{aligned}
\int d^{3} x & \left(\sum_{\phi} \frac{\delta \Gamma}{\delta \phi} \frac{\delta \Gamma}{\delta K_{\phi}}\right. \\
& \left.+b \frac{\delta \Gamma}{\delta \hat{c}}+i \bar{\zeta} \frac{\delta \Gamma}{\delta \overline{\hat{\varphi}}}+h \frac{\delta \Gamma}{\delta \hat{\omega}}\right)=0 .
\end{aligned}
$$

where the sum is extended over $\phi^{a}=\chi^{a}, V_{\mu}^{a}, H^{a}$, $\Lambda^{a}, c^{a}, \varphi^{a}, \omega^{a}$. In what follows, we will write this equation as $(\Gamma, \Gamma)=0$ and use the notation $\Theta$ for the Slavnov-Taylor operator: $\Theta=\left(\Gamma_{0},\right)$. An important property of $\Theta$ is that it commutes with the supersymmetry generator $\delta:[\Theta, \delta]=0$.

We remark that $\Gamma$ generates 1PI Green functions for the fields $V_{\mu}^{a}$ and $\Lambda^{a}$ and not for the elementary fields $A_{\mu}^{a}$ and $\lambda^{a}$. This is due to the fact that $S_{\mathrm{ES}}$ introduces external sources for the BRS variations of $V_{\mu}^{a}$ and $\Lambda^{a}$, and not for those of $A_{\mu}^{a}$ and $\lambda^{a}$. To compute $\Gamma$, we use the Feynman rules for $A_{\mu}^{a}$ and $\lambda^{a}$ and treat $V_{\mu}^{a}$ and $\Lambda$ as composite fields defined by eqs. (1) and (2). It is not difficult to see that, given a 1PI diagram with superficial degree of divergence $\bar{\omega}$, all the diagrams that result from replacing one or more of the external $A_{\mu}^{a}$ and/or $\lambda^{n}$-lines with any of the composite fields on the right-hand side in eqs. (1) and (2) have superficial degree of divergence strictly less than $\bar{\omega}$. Regarding then $V_{\mu}^{a}$ and $\Lambda^{a}$ as composite fields does not worsen power counting.

\section{Dimensional reduction and dimensional regularization}

In $\mathrm{DReD}$, all the fields and matrices are kept three-dimensional and the momenta are continued in the sense of ordinary DReG to $d<3$. Because the Dirac algebra is performed in three dimensions, the Fierz identities remain valid and DReD manifestly preserves supersymmetry. The regularized action computed with $\mathrm{DReD}$ satisfies then $\delta \Gamma^{\mathrm{DReD}}=0$. The BRS transformation for the gauge field in DReD, however, is not the same as in the unregularized theory. Indeed, whereas the first $d<3$ components of the gauge field have the same BRS transformation law as the gauge field in the unregularized theory, the last $3-d$ components transform as $s A_{\mu}^{a}=f^{a b c} A_{\mu}^{b} c^{c}$. Due to this fact, $\mathrm{DReD}$ does not manifestly preserve 
BRS invariance. It may happen that at the end of all calculations, once the limit $d \rightarrow 3$ has been taken, all effects due to the splitting of the gauge field into $d$ and $3-d$ components go away, but this is not what is meant by manifest BRS invariance. Concerning the well known algebraic inconsistency [5] that occurs in products of three or more epsilons in $\mathrm{DReD}$, we mention that it disappears in the limit $d \rightarrow 3$, since contributions with three or more epsilons are finite by power counting at $d=3$.

To define DReG, we follow ref. [6] and treat $\epsilon^{\mu \nu \rho}$ as purely three-dimensional [1]. This gives for the propagator of the gauge field in $d \geq 3 \mathrm{di}$ mensions

$\Delta_{\mu \nu}(p)=D_{\mu \nu}(p)+R_{\mu \nu}(p)$,

where

$$
\begin{array}{r}
D_{\mu \nu}(p)=-g^{2} m \frac{m \epsilon_{\mu \rho \nu} p^{\rho}+i p^{2} g_{\mu \nu}-i p_{\mu} p_{\nu}}{p^{2}\left(p^{2}+m^{2}-i o\right)} \\
R_{\mu \nu}(p)=-\frac{g^{2} m^{3}}{\left(p^{2}-i o\right)^{2}+m^{2} \tilde{p}^{2}}\left[\frac{1}{p^{2}+m^{2}-i o}\right. \\
\times \frac{\hat{p}^{2}}{p^{2}}\left(m \epsilon_{\mu \rho \nu} p^{\rho}+i p^{2} g_{\mu \nu}+\frac{i m^{2}}{p^{2}-i o} p_{\mu} p_{\nu}\right) \\
\left.+\frac{i}{p^{2}-i o}\left(\tilde{p}^{2} \hat{g}_{\mu \nu}-p_{\mu} \hat{p}_{\nu}-\hat{p}_{\mu} p_{\nu}+\hat{p}_{\mu} \hat{p}_{\nu}\right)\right]
\end{array}
$$

Here $g_{\mu \nu}$ and $p^{\mu}$ are $d$-dimensional, objects with a tilde are three-dimensional and objects with a caret are $(d-3)$-dimensional. Since the propagator is the inverse of the kinetic term in the $d$ dimensional classical action and the BRS transformation for the gauge field is the same as in the regularized theory, DReG preserves BRS invariance [6] [7]. Hence, the DReG regularized effective action satisfies the BRS identity $\left(\Gamma^{\mathrm{DReG}}, \Gamma^{\mathrm{DReG}}\right)=0$. The complicated propagator for the gauge field is the price for having a consistent treatment of $\epsilon^{\mu \nu \rho}$ while manifestly preserving BRS invariance. As regards supersymmetry, it is well known that DReG does not manifestly preserve it.

\section{Perturbative finiteness}

To prove perturbative finiteness at one loop, we consider a one-loop 1PI diagram and denote by
$\mathcal{D}(d)$ its value in DReG. According to eq. (3), if the diagram has an internal gauge line, $\mathcal{D}(d)$ is the sum of two contributions: $\mathcal{D}(d)=\mathcal{D}_{D}(d)+\mathcal{D}_{R}(d)$. The contribution $\mathcal{D}_{D}(d)$ contains the $S O(d)$ covariant part $D_{\mu \nu}$ of all the gauge propagators. The contribution $\mathcal{D}_{R}(d)$ contains at least one $R_{\mu \nu}$ and can be easily seen to be both UV and IR finite at $d=3$ by power counting. Being finite at $d=3$ and being at least linear in $\hat{g}_{\mu \nu}, \mathcal{D}_{R}(d)$ vanishes as $d \rightarrow 3$. We are thus left with only the $S O(d)$ covariant $\mathcal{D}_{D}(d)$. If the diagram has no internal gauge line, $\mathcal{D}(d)$ is already $S O(d)$ covariant. Using that $S O(d)$ covariant one-loop integrals have no poles when $d$ is continued to a positive odd integer [8] completes the proof at one loop. This also proves that in the limit $d \rightarrow 3,1 \mathrm{PI}$ Green functions at one loop are identical in DReG and in $\mathrm{DReD}$.

At two loops we proceed differently. Let us assume that the two-loop correction $\Gamma_{2}^{\mathrm{DReG}}$ to the effective action consists in the limit $d \rightarrow 3$ of a divergent part $\Gamma_{2 \text {,div }}^{\mathrm{DReG}}$ and a finite part $\Gamma_{2, \text { fin }}^{\mathrm{DReG}}$. Since $\Gamma_{2}^{\mathrm{DReG}}$ satisfies the BRS identity

$\Theta \Gamma_{2}^{\text {DReG }}+\left(\Gamma_{1}^{\text {DReG }}, \Gamma_{1}^{\text {DReG }}\right)=0$

and $\Gamma_{1}^{\mathrm{DReG}}$ is finite, the divergent part $\Gamma_{2 \text {,div }}^{\mathrm{DReG}}$ satisfies $\theta \Gamma_{2 \text { div }}^{\text {DReG }}=0$. Because 1PI Feynman diagrams with external sources as external lines are finite by power counting and there are no oneloop subdivergences, $\Gamma_{2 \text {,div }}^{\mathrm{DReG}}$ does not depend on the external sources and $\Theta \Gamma_{2, \text { div }}^{\mathrm{DReG}}=0$ reduces to $s \Gamma_{2, \text { div }}^{\mathrm{DReG}}=0$. Using the power counting in Table 1 and that contributions to two-loop 1PI diagrams from $R_{\mu \nu}$ are finite, we have that the most general form of $\Gamma_{2, \text { div }}^{\mathrm{DReG}}$ is $\Gamma_{2, \text { div }}^{\mathrm{DReG}}=\frac{1}{d-3} P_{\tilde{\omega}_{2}}$, where

$$
\begin{aligned}
P_{\bar{\omega}_{2}} & =m \int d^{3} x\left[\alpha_{1} m \bar{\chi}^{a} \chi^{a}+\alpha_{2} \bar{\chi}^{a} \not \partial \chi^{a}\right. \\
& +\alpha_{3} \overline{\chi^{a}} \lambda^{a}+\alpha_{4} A^{a} A^{a} \\
& +\alpha_{5} H^{a} H^{a}+\alpha_{6} f^{a b c} \bar{\chi}^{a} A^{b} \chi^{c} \\
& \left.+\alpha_{7} f^{a b c} f^{c d e}\left(\bar{\chi}^{a} \gamma^{\mu} \chi^{b}\right)\left(\bar{\chi}^{d} \gamma_{\mu} \chi^{e}\right)\right]
\end{aligned}
$$

and $\alpha_{1}, \ldots, \alpha_{7}$ are numerical coefficients. The equation $s \Gamma_{2, \text { div }}^{\mathrm{DReG}}=0$ is an equation in the coefficients $\alpha_{i}$ whose only solution is $\alpha_{i}=0$. This completes the proof at two loops.

The proof at three loops is analogous. Now 
the only three-loop Lorentz invariant divergence is $\Gamma_{3, d i v}^{\mathrm{DReG}}=\frac{1}{d-3} P_{\bar{\omega}_{3}}$, with

$P_{\bar{\omega}_{3}}=\alpha m^{2} \int d^{3} x \bar{\chi}^{a} \chi^{a}$,

but $P_{\bar{\omega}_{3}}$ is not BRS invariant. At higher loops, finiteness follows from power counting and from absence of subdivergences.

\section{A BRS invariant and supersymmetric effective action}

Since the theory is finite, every regularization method defines a renormalization scheme. We consider two renormalization schemes: scheme one uses DReG as regulator and performs no subtractions, scheme two uses $\mathrm{DReD}$ and performs no subtractions. We want to prove that the difference $\Delta \Gamma=\Gamma^{\mathrm{DReG}}-\Gamma^{\mathrm{DReD}}$ between the corresponding renormalized effective actions is zero. We have seen in section 4 that this is the case at one loop. So let us consider the two-loop case.

There is a general theorem in quantum field theory [9] that states that if two different renormalization (not regularization) schemes yield the same Green functions up to $k-1$ loops, then at $k$ loops they give Green functions that can differ at most by a local finite polynomial in the external momenta of degree equal to the superficial overall UV degree of divergence $\bar{\omega}_{k}$ at $k$ loops. This, and the power counting in Table 1 , implies that the difference $\Delta \Gamma_{2}$ at two loops can at most be of the form

$\Gamma_{2}^{\mathrm{DReG}}-\Gamma_{2}^{\mathrm{DReD}}=P_{\bar{\omega}_{2}}$,

with $P_{\bar{\omega}_{2}}$ as in eq. (5). We observe that, since $\mathrm{DReD}$ preserves supersymmetry, $\Gamma_{2}^{\mathrm{DReD}}$ satisfies

$\delta \Gamma_{2}^{\mathrm{DReD}}=0$.

Acting with $\delta$ on eq. (4), using eqs. (6) and (7), and recalling that $[\Theta, \delta]=0$ and that $\Delta \Gamma_{1}=0$, we obtain that $\Theta \delta P_{\bar{\omega}_{2}}=0$. Since $P_{\bar{\omega}_{2}}$ does not depend on the external sources, $\delta P_{\bar{\omega}_{2}}$ is independent of the external sources and $\Theta \delta P_{\bar{\omega}_{2}}=0$ reduces to $s \delta P_{\bar{\omega}_{2}}=0$, which is an equation in the coefficients $\alpha_{i}$ in $P_{\bar{\omega}_{2}}$. Because $\delta P_{\bar{\omega}_{2}}$ depends polynomially on the components of the gauge multiplet and their derivatives and has an overall factor of $m$, any nontrivial $\delta P_{\bar{\omega}_{2}}$ satisfying $s \delta P_{\bar{\omega}_{2}}=0$ must be $m$ times a local BRS invariant of mass dimension two. However, there are no such invariants. Hence, $\delta P_{\bar{\omega}_{2}}=0$. The only supersymmetry invariant that can be formed from $P_{\bar{\omega}_{2}}$ is

$$
\begin{aligned}
P_{\bar{\omega}_{2}}^{\text {susy }} & =\alpha m \int d^{3} x\left[\frac{1}{2} \bar{\chi}^{a} \not \partial \chi^{a}\right. \\
& +\bar{\chi}^{a} \lambda^{a}+A^{a} A^{a}-H^{a} H^{a} \\
& \left.-\frac{1}{48} f^{a b c} f^{c d e}\left(\bar{\chi}^{a} \gamma^{\mu} \chi^{b}\right)\left(\bar{\chi}^{d} \gamma_{\mu} \chi^{e}\right)\right] .
\end{aligned}
$$

At this point we have exhausted all the information given by BRS symmetry and supersymmetry. We determine the value of the coefficient $\alpha$ in $P_{\bar{\omega}_{2}}^{\text {susy }}$ by means of an explicit calculation (see below) and find $\alpha=0$.

At three loops, the difference is $\Delta \Gamma_{3}=\alpha P_{\bar{\omega}_{3}}$. Since $\Delta \Gamma_{3}$ is not BRS invariant, nor supersymmetric, the same arguments as used at the twoloop level are now powerful enough to conclude that $\alpha=0$ without the need of any explicit computation. At higher loops, the difference $\Delta \Gamma$ vanishes since at one, two and three loops it vanishes and there are no overall divergences by power counting.

We now compute $\alpha$ in $P_{\bar{\omega}_{2}}^{\text {susy }}$. To do this, we evaluate the difference between the contributions from DReG and DReD to the selfenergy of the field $H^{a}$. The vertices with an $H$ are $H \zeta \chi, H \hat{\varphi} \varphi, H \hat{\omega} c$ and $H \hat{\varphi} \chi c$. Using them, one can construct two-loop 1PI diagrams with the six topologies in Fig. 1. In fact, since $\hat{\varphi}$ only propagates in $\varphi$ and $c$ into $\hat{c}$, and there is no four-point vertex containing the fields $H, \varphi$ and $\hat{c}$, no graphs with the topology of Fig. 1a can be constructed. The topologies in Figs. 1b and 1c, being products of one-loop topologies, give the same contributions in DReG as in DReD, hence they do not contribute to $\alpha$. We are thus left with the topologies in Figs. 1d, 1e and 1f. Because one-loop subdiagrams give the same contributions in DReG as in $\mathrm{DReD}$, only the overall divergent part of the corresponding two-loop diagrams contribute to $\alpha$. Since the two-loop diagrams are logarithmically divergent, the contributions to $\alpha$ come from setting in the numerators the external momentum $p^{\mu}$ and the mass $m$ equal to zero, except, of course, for the overall factor $m$. The overall 


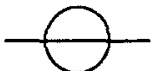

(a)

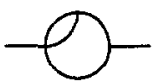

(d)

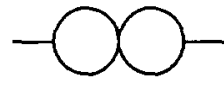

(b)

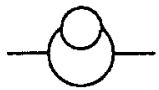

(e)

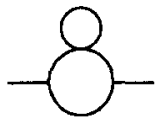

(c)

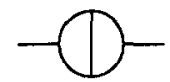

(f)
Figure 1: Two-loop topologies for $\langle H H\rangle_{1 \mathrm{PI}}$

divergent part of every diagram then reads

$m \int \frac{d^{d} k}{(2 \pi)^{d}} \frac{d^{d} q}{(2 \pi)^{d}} \frac{N(k, q)}{D(k, q, p, m)}$.

It is very easy to see that the numerator $N(k, q)$ always contains a trace over a fermion loop. This, and the observation that diagrams with internal gauge lines only occur in topology $1 \mathrm{e}$ and that their contributions separately cancel, implies that the overall divergence in DReG and DReD are the same except for the trace over the fermions. The trace of a sum of products of $\phi$ and $k$ can always be written as $d$-dimensional scalar products $k^{2}, k q$ and $q^{2}$ times an overall trace of the unit matrix. After summing over diagrams, $\alpha$ can then be written as

$$
\begin{aligned}
\alpha & =\left(\operatorname{tr}_{\mathrm{DReG}} \mathbb{1}-\operatorname{tr}_{\mathrm{DReD}} \mathbb{1}\right) \\
& \times \int \frac{d^{d} k}{(2 \pi)^{d}} \frac{d^{d} q}{(2 \pi)^{d}} \frac{f\left(k^{2}, k q, q^{2}\right)}{D(k, q, p, m)},
\end{aligned}
$$

where $f\left(k^{2}, k q, q^{2}\right)$ is a polynomial of its arguments. Because the theory is finite, the integral is finite and therefore the difference due to the trace vanishes in the limit $d \rightarrow 3$. Hence $\alpha=0$.

The equality of $\Gamma^{\mathrm{DReG}}$ and $\Gamma^{\mathrm{DReD}}$ is not explained by local quantum field theory. One possible explanation might be that there exists a third, as yet unknown, symmetry of the model. Another explanation might be that the existing theorems of local quantum field theory [9] concerning the difference between the renormalized expressions for the same Green function computed in two different renormalization schemes can be sharpened for finite models which are superrenormalizable by power counting and which have symmetries.

Our analysis relies on the fact that our model is superrenormalizable by power counting and finite. There exist several all-loop [10] finite supersymmetric models in four dimensions, and $N=4$
Yang-Mills theory is also all-loop finite. It would be interesting to apply the methods developed in this paper to these models (see ref. [11] for a partial comparison of DReG and DReD in 4dimensional $N=1$ Yang-Mills theory in a nonsupersymmetric gauge).

\section{REFERENCES}

1. G. 't Hooft and M. Veltman, Nucl Phys. B44 (1972) 189.

2. W. Siegel, Phys. Lett. 84B (1979) 193.

3. F. Ruiz Ruiz and P. van Nieuwenhuizen, $B R S$ symmetry versus supersymmetry in YangMills-Chern-Simons theory, to appear in Nucl. Phys. B (hep-th/9609074).

4. S.J. Gates Jr., M.T. Grisaru, M. Roček and W. Siegel, Superspace or one thousand and one lessons in supersymmetry (Benjamin, Reading 1983).

W. Siegel, Nucl. Phys. B156 (1979) 135.

5. W. Siegel, Phys. Lett. 94B (1980) 37.

6. G. Giavarini, C.P. Martin and F. Ruiz Ruiz, Nucl. Phys. B381 (1992) 222; Phys. Rev. D47 (1993) 5536; Phys. Lett. B314 (1993) 328; Phys. Lett. B332 (1994) 345.

7. P. Breitenlohner and D. Maison, Commun. Math. Phys. 52 (1977) 11.

8. E.R. Speer, J. Math. Phys. 15 (1974) 1; Ann. Inst. Henri Poincaré XXII (1975) 1.

9. K. Hepp, Renormalization theory, in Statistical Mechanics and quantum field theory, edited by C. DeWitt and R. Stora (Gordon and Breach, New York 1971).

H. Epstein and V. Glasser, Ann. Inst. Henri Poincaré XIX (1973) 211.

10. A.V. Ermushev, D.I. Kazakov and O.V. Tarasov, Nucl. Phys. B281 (1987) 72.

D.I. Kazakov, Phys. Lett. B179 (1986) 952.

C. Lucchesi, O. Piguet and K. Sibold, Helv. Phys. Acta 61 (1988) 321 and Phys. Lett. B201 (1988) 241.

C. Lucchesi, Finiteness in $N=1 S Y M$ theories (hep-ph/9607368).

11. D.M. Capper, D.R.T. Jones and P. van Nieuwenhuizen, Nucl. Phys. B167 (1980) 479. 